PROCEEDINGS OF THE

AMERICAN MATHEMATICAL SOCIETY

Volume 132, Number 7, Pages 2075-2083

S 0002-9939(03)07329-5

Article electronically published on December 31, 2003

\title{
LOGARITHMIC SOBOLEV TRACE INEQUALITY
}

\author{
YOUNG JA PARK
}

(Communicated by Andreas Seeger)

\begin{abstract}
A logarithmic Sobolev trace inequality is derived. Bounds on the best constant for this inequality from above and below are investigated using the sharp Sobolev inequality and the sharp logarithmic Sobolev inequality.
\end{abstract}

Logarithmic Sobolev inequalities capture the spirit of classical Sobolev inequalities with the logarithm function replacing powers, and they can be considered as limiting cases of the classical Sobolev inequalities.

In the original analysis of the logarithmic Sobolev inequality, Gross 13 emphasized its infinite-dimensional character and the dimension-independent nature of its constants. Recent arguments by Beckner [7, 8, 9] used the product structure of the domain and asymptotics for the sharp Sobolev embedding to derive the logarithmic Sobolev inequality with more explicit geometric character: for a smooth function $f \in \mathbf{S}\left(\mathbb{R}^{n}\right)$ with $\|f\|_{L^{2}\left(\mathbb{R}^{n}\right)}=1$,

$$
\int_{\mathbb{R}^{n}}|f(x)|^{2} \ln |f(x)| d x \leq \frac{n}{4} \ln \left[\frac{2}{\pi e n} \int_{\mathbb{R}^{n}}|\nabla f(x)|^{2} d x\right],
$$

where $\mathbf{S}\left(\mathbb{R}^{n}\right)$ represents the Schwartz class of functions on $\mathbb{R}^{n}$. It has been recognized by E. Carlen [10] that logarithmic Sobolev inequalities are direct consequences of strict superadditivity of the Fisher information.

The sharp Sobolev trace inequality for $n>1$ in [4] is

$$
\left(\int_{\mathbb{R}^{n}}|f(x)|^{2 n /(n-1)} d x\right)^{\frac{n-1}{n}} \leq \frac{1}{\sqrt{\pi}} \frac{1}{n-1}\left[\frac{\Gamma(n)}{\Gamma(n / 2)}\right]^{\frac{1}{n}} \int_{\mathbb{R}_{+}^{n+1}}|\nabla v(x, y)|^{2} d x d y,
$$

where $v$ is a harmonic extension of $f$ to the upper half-space that is continuous on the closed upper half-space. Beckner proved this by inverting the inequality to a fractional integral on the dual space and using a special case of the sharp Hardy-Littlewood-Sobolev inequality. Independently, J. Escobar [11] proved this by exploiting the conformal invariance of the variational equation and using characteristics of an Einstein metric. This result was used to answer the Yamabe problem on a Riemannian manifold with boundary [12].

Received by the editors April 18, 2001 and, in revised form, July 1, 2001, April 19, 2002, and April 9, 2003

2000 Mathematics Subject Classification. Primary 46E35, 42C99.

Key words and phrases. Sobolev trace inequalities, logarithmic Sobolev inequalities, logarithmic uncertainty principle. 
In section 1, a logarithmic Sobolev trace inequality will be derived: for $f \in \mathbf{S}\left(\mathbb{R}^{n}\right)$ with $\|f\|_{L^{2}\left(\mathbb{R}^{n}\right)}=1$ and $n>1$,

$$
\int_{\mathbb{R}^{n}}|f(x)|^{2} \ln |f(x)| d x \leq \frac{n}{2} \ln \left[A_{n} \int_{\mathbb{R}_{+}^{n+1}}|\nabla u(x, y)|^{2} d x d y\right],
$$

where $u$ is an extension of $f$ to the upper half-space that is continuous in the closed upper half-space and at least once differentiable on the open upper half-space, and $A_{n}$ is a positive constant independent of $u$. It is clear that only harmonic extensions of $f$ are necessary to consider due to Dirichlet's principle. As in the case of the logarithmic Sobolev inequalities [8], the logarithmic Sobolev trace inequality will be obtained as a limiting case of the Sobolev trace inequalities.

In section 2, the best or optimal constant $\mathbf{E}_{\mathbf{n}}$ for the logarithmic Sobolev trace inequality will be defined. The objective of this section is to get bounds on $\mathbf{E}_{\mathbf{n}}$ from above and below. By using information on the best constant for the logarithmic Sobolev inequality, it will be proved that for $n>1$,

$$
\sqrt{\pi}(n-1)[\Gamma(n / 2) / \Gamma(n)]^{\frac{1}{n}} \leq \mathbf{E}_{\mathbf{n}} \leq \sqrt{\pi e n / 2} .
$$

Thus for large $n$, one finds the asymptotic estimate

$$
\mathbf{E}_{\mathbf{n}} \simeq \sqrt{\pi e n / 2} .
$$

In section 3 , the constant $E_{n}\left(e^{-\pi|x|^{2}}\right)$ will be compared to get a better upper bound for $\mathbf{E}_{\mathbf{n}}$ with $\sqrt{\frac{\pi e n}{2}}$, the best constant for the logarithmic Sobolev inequality.

In section 4, a logarithmic Sobolev trace inequality will be derived using the logarithmic uncertainty principle, which was proved by Beckner in [6]. This covers the case $n=1$, which is missed in the derivation in section 1 .

In section 5, a rearrangement technique will be introduced to reduce the functions to considering when one wants to find the best constant. It turns out that it suffices to only consider radial decreasing functions on $\mathbb{R}^{n}$.

\section{Logarithmic Sobolev trace inequality}

A logarithmic Sobolev trace inequality will be derived from the sharp Sobolev trace inequality, and by doing so one can recognize it as a limiting case of the classical Sobolev trace inequalities.

Theorem 1. For $f \in \mathbf{S}\left(\mathbb{R}^{n}\right)$ with $\|f\|_{L^{2}\left(\mathbb{R}^{n}\right)}=1$ and $n>1$,

$$
\int_{\mathbb{R}^{n}}|f(x)|^{2} \ln |f(x)| d x \leq \frac{n}{2} \ln \left(A_{n} \int_{\mathbb{R}_{+}^{n+1}}|\nabla u(x, y)|^{2} d x d y\right),
$$

where $u$ is an extension of $f$ to the upper half-space that is continuous in the closed upper half-space and at least once differentiable on the open upper half-space, and $A_{n}=\frac{1}{\sqrt{\pi}} \frac{1}{n-1}[\Gamma(n) / \Gamma(n / 2)]^{1 / n}$.

Proof. Recall the sharp Sobolev trace inequality in [4]: for $f \in \mathbf{S}\left(\mathbb{R}^{n}\right)$ with $\|f\|_{L^{2}\left(\mathbb{R}^{n}\right)}$ $=1$ and $n>1$,

$$
\left(\int_{\mathbb{R}^{n}}|f(x)|^{2 n /(n-1)} d x\right)^{(n-1) / n} \leq A_{n} \int_{\mathbb{R}_{+}^{n+1}}|\nabla v(x, y)|^{2} d x d y,
$$


where $v$ is a harmonic extension of $f$ to the upper half-space that is continuous in the closed upper half-space. With the assumption $\|f\|_{L^{2}\left(\mathbb{R}^{n}\right)}=1$ by which $|f(x)|^{2} d x$ can be thought of as a probability measure, one can obtain

$$
\lim _{r \rightarrow 2}\left(\int_{\mathbb{R}^{n}}|f(x)|^{r-2}|f(x)|^{2} d x\right)^{1 /(r-2)}=\exp \left(\int_{\mathbb{R}^{n}}|f(x)|^{2} \ln |f(x)| d x\right) .
$$

It is from applying Hölder's inequality and the sharp Sobolev trace inequality (4) that for $f \in \mathbf{S}\left(\mathbb{R}^{n}\right)$ with $\|f\|_{L^{2}\left(\mathbb{R}^{n}\right)}=1$ and for $2<r<\frac{2 n}{n-1}$,

$$
\begin{aligned}
\left(\int_{\mathbb{R}^{n}}|f(x)|^{r} d x\right)^{\frac{1}{r-2}} & =\left(\int_{\mathbb{R}^{n}}|f(x)|^{n(r-2)}|f(x)|^{2 n-(n-1) r} d x\right)^{\frac{1}{r-2}} \\
& \leq\left(\int_{\mathbb{R}^{n}}|f(x)|^{\frac{2 n}{n-1}} d x\right)^{\frac{n-1}{2}}\left(\int_{\mathbb{R}^{n}}|f(x)|^{2} d x\right)^{\frac{2 n-r n+r}{2(r-2)}} \\
& \leq\left(A_{n} \int_{\mathbb{R}_{+}^{n+1}}|\nabla v(x, y)|^{2} d x d y\right)^{\frac{n}{2}} .
\end{aligned}
$$

Taking the limit of both sides in (5) as $r$ approaches 2 from the right yields

$$
\exp \left(\int_{\mathbb{R}^{n}}|f(x)|^{2} \ln |f(x)| d x\right) \leq\left(A_{n} \int_{\mathbb{R}_{+}^{n+1}}|\nabla v(x, y)|^{2} d x d y\right)^{\frac{n}{2}} .
$$

Dirichlet's principle makes this argument yield the desired logarithmic Sobolev trace inequality.

\section{Definition and some estimates of the Best constant $\mathbf{E}_{\mathbf{n}}$}

It is well known that sharp constants contain geometric and probabilistic information of spaces (1], 2], [4, [7]). In this aspect, it will be interesting to look at the sharp constant for the logarithmic Sobolev trace inequality. In this section, the best constant for the logarithmic Sobolev trace inequality $\mathbf{E}_{\mathbf{n}}$ will be defined. The goal of this section is to find estimates of $\mathbf{E}_{\mathbf{n}}$ from above and below. In fact, those bounds can be found by investigating the sharp logarithmic Sobolev inequality and the sharp Sobolev inequality.

Let $f \in \mathbf{S}\left(\mathbb{R}^{n}\right)$ with $\|f\|_{L^{2}\left(\mathbb{R}^{n}\right)}=1$ and let $u$ be an extension of $f$ to the upper half-space which is in $C\left(\overline{\mathbb{R}_{+}^{n+1}}\right) \cap C^{1}\left(\mathbb{R}_{+}^{n+1}\right)$. Consider the following quantity:

$$
E_{n}(f: u) \equiv \frac{\int_{\mathbb{R}_{+}^{n+1}}|\nabla u(x, y)|^{2} d x d y}{\exp \left[\frac{2}{n} \int_{\mathbb{R}^{n}}|f(x)|^{2} \ln |f(x)| d x\right]} .
$$

Definition 1. Define

$$
\mathbf{E}_{\mathbf{n}}=\inf \left\{E_{n}(f):\|f\|_{L^{2}\left(\mathbb{R}^{n}\right)}=1, f \in \mathbf{S}\left(\mathbb{R}^{n}\right)\right\},
$$

where $E_{n}(f)=\inf \left\{E_{n}(f: w): w(x, 0)=f(x), w \in C\left(\overline{\mathbb{R}_{+}^{n+1}}\right) \cap C^{1}\left(\mathbb{R}_{+}^{n+1}\right)\right\}$.

$\mathbf{E}_{\mathbf{n}}$ is called the best (sharp) constant for the logarithmic Sobolev trace inequality. It is easy to see from Theorem 1 that $A_{n}$ gives a lower bound for $\mathbf{E}_{\mathbf{n}}$ since $1 / A_{n} \leq \mathbf{E}_{\mathbf{n}}$, which yields the following: for $n>1$,

$$
\sqrt{\pi}(n-1)[\Gamma(n / 2) / \Gamma(n)]^{1 / n} \leq \mathbf{E}_{\mathbf{n}} .
$$


Dirichlet's principle and the Steiner symmetrization ([15, [16]) can be used to reduce the extension functions to considering when one wants to find the best constant since

$$
\int_{\mathbb{R}_{+}^{n+1}}\left|\nabla u_{S}(x, y)\right|^{2} d x d y \leq \int_{\mathbb{R}_{+}^{n+1}}|\nabla u(x, y)|^{2} d x d y,
$$

where $u_{S}$ represents the Steiner symmetrization of $u$ with respect to the direction of $e_{n+1}=(0, \ldots, 0,1)$. Since harmonic extensions of $f$ that are decreasing functions of $y$ give the smallest numerator of $E_{n}(f: \cdot)$ when $f$ is fixed, one has

$$
\begin{aligned}
E_{n}(f)= & \inf \left\{E_{n}(f: w): w(x, 0)=f(x), w \in C\left(\overline{\mathbb{R}_{+}^{n+1}}\right) \cap C^{1}\left(\mathbb{R}_{+}^{n+1}\right)\right\} \\
= & \inf \left\{E_{n}(f: v): v(x, 0)=f(x), \Delta v=0 \text { on } \mathbb{R}_{+}^{n+1}, v \in C\left(\mathbb{R}_{+}^{n+1}\right),\right. \text { and } \\
& v(x, \cdot) \text { is a decreasing function of } y\} .
\end{aligned}
$$

Beckner [9] proved the following sharp logarithmic Sobolev inequality: for $f \in$ $\mathbf{S}\left(\mathbb{R}^{n}\right)$ with $\|f\|_{L^{2}\left(\mathbb{R}^{n}\right)}=1$,

$$
\int_{\mathbb{R}^{n}}|f(x)|^{2} \ln |f(x)| d x \leq \frac{n}{4}\left[\ln \frac{2}{\pi e n} \int_{\mathbb{R}^{n}}|\nabla f(x)|^{2} d x\right],
$$

and the best constant is attained for the Gaussian functions. See also the earlier arguments by Stam [17] and Weissler [20]. This can be put into the following context: for $f \in \mathbf{S}\left(\mathbb{R}^{n}\right)$ with $\|f\|_{L^{2}\left(\mathbb{R}^{n}\right)}=1$, define

$$
\mathbf{T}_{\mathbf{n}}=\inf \left\{T_{n}(f)=\frac{\int_{\mathbb{R}^{n}}|\nabla f(x)|^{2} d x}{\exp \left[\frac{4}{n} \int_{\mathbb{R}^{n}}|f(x)|^{2} \ln |f(x)| d x\right]}:\|f\|_{L^{2}\left(\mathbb{R}^{n}\right)}=1, f \in \mathbf{S}\left(\mathbb{R}^{n}\right)\right\},
$$

and then one has $\mathbf{T}_{\mathbf{n}}=\pi e n / 2$.

Now, compare $T_{n}(f)$ with $E_{n}(f)$. It is well known [19] that when $v$ is a harmonic extension of $f$ to the upper half-space and $v(x, \cdot)$ is a decreasing function of $y$, $\|v(\cdot, y)\|_{L^{2}\left(\mathbb{R}^{n}\right)}$ is uniformly bounded by $\|v(\cdot, 0)\|_{L^{2}\left(\mathbb{R}^{n}\right)}$ for all $y>0, v$ is the Poisson integral of $f$ and

$$
\int_{\mathbb{R}_{+}^{n+1}}|\nabla v(x, y)|^{2} d x d y=2 \pi \int_{\mathbb{R}^{n}}|\xi||\hat{f}(\xi)|^{2} d \xi,
$$

where $\hat{f}$ denotes the Fourier transform of $f$ defined by $\hat{f}(\xi)=\int_{\mathbb{R}^{n}} f(x) e^{2 \pi i x \cdot \xi} d x$. Since $\|f\|_{L^{2}\left(\mathbb{R}^{n}\right)}=1$ is assumed, one also has $\|\hat{f}\|_{L^{2}\left(\mathbb{R}^{n}\right)}=1$. From this one now has

$$
E_{n}(f)=\frac{2 \pi \int_{\mathbb{R}^{n}}|\xi||\hat{f}(\xi)|^{2} d \xi}{\exp \left(\frac{2}{n} \int_{\mathbb{R}^{n}}|f(x)|^{2} \ln |f(x)| d x\right)} .
$$

The Plancherel formula together with Jensen's inequality yields

$$
4 \pi^{2}\left[\int_{\mathbb{R}^{n}}|\xi||\hat{f}(\xi)|^{2} d \xi\right]^{2} \leq 4 \pi^{2} \int_{\mathbb{R}^{n}}|\xi|^{2}|\hat{f}(\xi)|^{2} d \xi=\int_{\mathbb{R}^{n}}|\nabla f(x)|^{2} d x .
$$

Then,

$$
\left[E_{n}(f)\right]^{2} \leq \frac{\int_{\mathbb{R}^{n}}|\nabla f(x)|^{2} d x}{\exp \left[\frac{4}{n} \int_{\mathbb{R}^{n}}|f(x)|^{2} \ln |f(x)| d x\right]} .
$$

It follows from (8) that for any $f \in \mathbf{S}\left(\mathbb{R}^{n}\right), E_{n}(f) \leq \sqrt{T_{n}(f)}$, which implies

$$
\mathbf{E}_{\mathbf{n}} \leq \sqrt{\pi e n / 2}=\sqrt{\mathbf{T}_{\mathbf{n}}} .
$$




\section{Comparison: Better upper Bound}

$E_{n}\left(e^{-\pi|x|^{2}}\right)$ will be considered, and it will be shown that the Gaussian function $e^{-\pi|x|^{2}}$ gives a better upper bound for the logarithmic Sobolev trace inequality through a comparison between $E_{n}\left(e^{-\pi|x|^{2}}\right)$ and the upper bound $\mathbf{T}_{\mathbf{n}}=\sqrt{\pi e n / 2}$ given in the previous section.

Using (77) it can be easily computed that

$$
E_{n}\left(e^{-\pi|x|^{2}}\right)=\sqrt{\pi e} \frac{\Gamma\left(\frac{n+1}{2}\right)}{\Gamma\left(\frac{n}{2}\right)} .
$$

For a comparison, take the ratio of the two constants

$$
g(n) \equiv \frac{E_{n}\left(e^{-\pi|x|^{2}}\right)}{\mathbf{T}_{\mathbf{n}}}=\sqrt{\frac{2}{n}} \frac{\Gamma\left(\frac{n+1}{2}\right)}{\Gamma\left(\frac{n}{2}\right)} .
$$

For any integer $n \geq 1, g(n)$ can be rewritten as

$$
g(n)= \begin{cases}\sqrt{\frac{\pi}{m} \frac{1 \cdot 3 \cdots(2 m-3)(2 m-1)}{2^{m}(m-1) !}} & \text { if } n=2 m, \\ \sqrt{\frac{2}{\pi(2 m-1)} \frac{2^{m}(m-1) !}{1 \cdot 3 \cdots(2 m-5)(2 m-3)}} & \text { if } n=2 m-1 .\end{cases}
$$

It is easy to see that

$$
\frac{g(2 m)}{g(2 m+2)}=\sqrt{\frac{4 m(m+1)}{(2 m+1)^{2}}}<1, \quad \frac{g(2 m-1)}{g(2 m+1)}=\sqrt{\frac{(2 m-1)(2 m+1)}{4 m^{2}}}<1,
$$

which means

$$
g(2 m)<g(2 m+2) \text { and } g(2 m-1)<g(2 m+1) \text { for } m=1,2,3, \cdots .
$$

Stirling's formula can be used to show that

$$
\lim _{n \rightarrow \infty} g(n)=1 .
$$

Putting (9) and (10) together gives

$$
g(n)<1 \text { for any } n=1,2,3, \cdots .
$$

In fact, otherwise, there would exist an integer $n_{0}$ such that $g\left(n_{0}\right) \geq 1$. Then by (91), $g\left(n_{0}+2\right)>1$, which contradicts the fact that the subsequences $\{g(2 m)\}$ and $\{g(2 m-1)\}$ strictly increase to converge to 1 . This argument gives

$$
\mathbf{E}_{\mathbf{n}} \leq E_{n}\left(e^{-\pi|x|^{2}}\right)<\sqrt{\pi e n / 2}
$$

\section{Logarithmic Sobolev trace inequality Revisited}

A logarithmic Sobolev trace inequality will be derived from the logarithmic uncertainty principle. It should be noted that this derivation holds for any $n \geq 1$.

The derivation of a logarithmic Sobolev trace inequality from the logarithmic uncertainty principle is very simple. The logarithmic uncertainty principle in [6] is given by: for $f \in \mathbf{S}\left(\mathbb{R}^{n}\right)$ with $\|f\|_{L^{2}}=1$,

$$
\int_{\mathbb{R}^{n}}|f(x)|^{2} \ln |f(x)| d x+B_{n} \leq \frac{n}{2} \int_{\mathbb{R}^{n}}|\hat{f}(\xi)|^{2} \ln |\xi| d \xi,
$$

where $B_{n}=\frac{n}{2} \psi\left(\frac{n}{2}\right)-\frac{n}{4} \ln \pi-\frac{1}{2} \ln \left[\Gamma(n) / \Gamma\left(\frac{n}{2}\right)\right]$ and $\psi(t)=\frac{d}{d t}[\ln \Gamma(t)]$. Up to conformal automorphism, extremal functions are of the form $\left(1+|x|^{2}\right)^{-n / 2}$. Suppose 
$v$ is a harmonic extension of $f$ to the upper half-space. The concavity of the logarithm function can be combined with (6) and (11) to yield

$$
\int_{\mathbb{R}^{n}}|f(x)|^{2} \ln |f(x)| d x \leq \frac{n}{2} \ln \left[\frac{1}{2 \pi} e^{-\frac{2}{n} B_{n}} \int_{\mathbb{R}_{+}^{n+1}}|\nabla v(x, y)|^{2} d x d y\right] .
$$

Dirichlet's principle leads to the logarithmic Sobolev trace inequality with $v$ replaced by an arbitrary extension of $f$.

For $f \in \mathbf{S}\left(\mathbb{R}^{n}\right)$ with $\|f\|_{L^{2}\left(\mathbb{R}^{n}\right)}=1$,

$$
\int_{\mathbb{R}^{n}}|f(x)|^{2} \ln |f(x)| d x \leq \frac{n}{2} \ln \left[e^{-\frac{2}{n} B_{n}} \int_{\mathbb{R}^{n}}|\xi||\hat{f}(\xi)|^{2} d \xi\right] .
$$

This form clearly brings out the intrinsic nature of a logarithmic Sobolev trace inequality as a comparison between the entropy of a function and a measure of its smoothness. The right-hand side of (12) can also be viewed as a measure of the concentration of $|\hat{f}|^{2}$ in a neighborhood of the origin, a small value corresponding to a high degree of concentration. The left-hand side of (12), being the entropy of $|f|^{2}$, is a measure of the concentration of $|f|^{2}$ on a set of small measure, a large positive value corresponding to a high degree of concentration. For the best constant for the logarithmic Sobolev trace inequalities, it only makes sense to consider functions that are concentrated on a set of small measure and whose Fourier transforms are also concentrated near the origin.

\section{REARRANGEMENT OF FUnCtions}

It will be proved that a rearrangement technique can be used to reduce the functions to considering when it comes to finding the best constant for the logarithmic Sobolev trace inequality.

When one wants to search for the best constant for the logarithmic Sobolev trace inequality, one looks at the quantity $E_{n}(f)$ (see (7)) for $f \in \mathbf{S}\left(\mathbb{R}^{n}\right)$ with $\|f\|_{L^{2}\left(\mathbb{R}^{n}\right)}=1$. Let $f^{*}$ denote the equimeasurable radial decreasing rearrangement of $|f|$ on $\mathbb{R}^{n}$, and then $\hat{f}^{*}$ will be the Fourier transform of $f^{*}$. It suffices to consider nonnegative radial decreasing functions for the best constant for the logarithmic Sobolev trace inequality if one can show

$$
\int_{\mathbb{R}^{n}}|\xi|\left|\hat{f}^{*}(\xi)\right|^{2} d \xi \leq \int_{\mathbb{R}^{n}}|\xi||\hat{f}(\xi)|^{2} d \xi,
$$

since it is known that

$$
\int_{\mathbb{R}^{n}}\left|f^{*}(x)\right|^{2} \ln \left|f^{*}(x)\right| d x=\int_{\mathbb{R}^{n}}|f(x)|^{2} \ln |f(x)| d x .
$$

Instead of (13), the following stronger version will be proved:

$$
\int_{\mathbb{R}^{n}}|\xi|^{\alpha}\left|\hat{f}^{*}(\xi)\right|^{2} d \xi \leq \int_{\mathbb{R}^{n}}|\xi|^{\alpha}|\hat{f}(\xi)|^{2} d \xi \quad \text { for } 0<\alpha<2 .
$$

\section{Lemma 1.}

$$
\int_{\mathbb{R}^{n}}\left(\frac{|\xi|^{2}}{1+|\xi|^{2}}\right)^{\alpha / 2}\left|\hat{f}^{*}(\xi)\right|^{2} d \xi \leq \int_{\mathbb{R}^{n}}\left(\frac{|\xi|^{2}}{1+|\xi|^{2}}\right)^{\alpha / 2}|\hat{f}(\xi)|^{2} d \xi
$$


Suppose that Lemma 1 has been proved. Take $\varepsilon>0$, and replace $f(x)$ by $f(\varepsilon x)$. It is true that $[f(\varepsilon x)]^{*}=f^{*}(\varepsilon x)$, since the radial decreasing rearrangement commutes with uniform dilation. Then, (15) becomes

$$
\frac{1}{\varepsilon^{2 n}} \int_{\mathbb{R}^{n}}\left(\frac{|\xi|^{2}}{1+|\xi|^{2}}\right)^{\alpha / 2}\left|\hat{f}^{*}(\xi / \varepsilon)\right|^{2} d \xi \leq \frac{1}{\varepsilon^{2 n}} \int_{\mathbb{R}^{n}}\left(\frac{|\xi|^{2}}{1+|\xi|^{2}}\right)^{\alpha / 2}|\hat{f}(\xi / \varepsilon)|^{2} d \xi
$$

Changing variables $\xi / \varepsilon=\eta$ gives

$$
\int_{\mathbb{R}^{n}}\left(\frac{|\eta|^{2}}{1+\varepsilon^{2}|\eta|^{2}}\right)^{\alpha / 2}\left|\hat{f}^{*}(\eta)\right|^{2} d \eta \leq \int_{\mathbb{R}^{n}}\left(\frac{|\eta|^{2}}{1+\varepsilon^{2}|\eta|^{2}}\right)^{\alpha / 2}|\hat{f}(\eta)|^{2} d \eta
$$

Take the limit of both sides as $\varepsilon \rightarrow 0$ to get (14).

Proof of Lemma 1 . Set $\alpha / 2=\beta$. Then $0<\beta<1$. With the following expression,

$$
\left(\frac{|\xi|^{2}}{1+|\xi|^{2}}\right)^{\beta}=\left(1-\frac{1}{1+|\xi|^{2}}\right)^{\beta}=1-\sum_{k=1}^{\infty}(-1)^{k+1}\left(\begin{array}{c}
\beta \\
k
\end{array}\right)\left(\frac{1}{1+|\xi|^{2}}\right)^{k}
$$

(15) now becomes

$$
\begin{aligned}
& \int_{\mathbb{R}^{n}}\left|\hat{f^{*}}(\xi)\right|^{2} d \xi-\sum_{k=1}^{\infty}(-1)^{k+1}\left(\begin{array}{c}
\beta \\
k
\end{array}\right) \int_{\mathbb{R}^{n}} \frac{1}{\left(1+|\xi|^{2}\right)^{k}}\left|\hat{f}^{*}(\xi)\right|^{2} d \xi \\
& \leq \int_{\mathbb{R}^{n}}|\hat{f}(\xi)|^{2} d \xi-\sum_{k=1}^{\infty}(-1)^{k+1}\left(\begin{array}{c}
\beta \\
k
\end{array}\right) \int_{\mathbb{R}^{n}} \frac{1}{\left(1+|\xi|^{2}\right)^{k}}|\hat{f}(\xi)|^{2} d \xi .
\end{aligned}
$$

It suffices to show that for each positive integer $k$,

$$
\int_{\mathbb{R}^{n}} \frac{1}{\left(1+|\xi|^{2}\right)^{k}}\left|\hat{f}^{*}(\xi)\right|^{2} d \xi \geq \int_{\mathbb{R}^{n}} \frac{1}{\left(1+|\xi|^{2}\right)^{k}}|\hat{f}(\xi)|^{2} d \xi
$$

since $(-1)^{k+1}\left(\begin{array}{c}\beta \\ k\end{array}\right)>0$ when $0<\beta<1$ and $k$ is any positive integer. Consider a function $g_{k}$ such that $\hat{g_{k}}(\xi)=\left(1+|\xi|^{2}\right)^{-k / 2}$, where $\tilde{g}(x)=\overline{g(-x)}$. In fact, when $G_{k}(x)$ is taken as

$$
G_{k}(x)=\frac{1}{\Gamma(k / 2)} \frac{1}{(4 \pi)^{k / 2}} \int_{0}^{\infty} e^{-\frac{\pi|x|^{2}}{\delta}} e^{-\frac{\delta}{4 \pi}} \delta^{-\frac{(n-k)}{2}} \frac{d \delta}{\delta},
$$

it can be shown that $\hat{G}_{k}(\xi)=\left(1+4 \pi^{2}|\xi|^{2}\right)^{-k / 2}$ (see [18]). Then one has

$$
\begin{aligned}
\int_{\mathbb{R}^{n}} \frac{1}{\left(1+|\xi|^{2}\right)^{k}}|\hat{f}(\xi)|^{2} d \xi & =(2 \pi)^{k} \int_{\mathbb{R}^{n}} \hat{G}_{2 k}(2 \pi \xi) \hat{f}(\xi) \overline{\hat{f}(\xi)} d \xi \\
& =(2 \pi)^{k} \int_{\mathbb{R}^{n}} G_{2 k}(-2 \pi x)(f * \tilde{f})(x) d x \\
& =(2 \pi)^{k} \int_{\mathbb{R}^{n} \times \mathbb{R}^{n}} G_{2 k}[2 \pi(y-z)] f(z) \overline{f(y)} d y d z \\
& \leq(2 \pi)^{k} \int_{\mathbb{R}^{n} \times \mathbb{R}^{n}} G_{2 k}[2 \pi(y-z)] f^{*}(z) \overline{f^{*}(y)} d y d z \\
& =\int_{\mathbb{R}^{n}} \frac{1}{\left(1+|\xi|^{2}\right)^{k}}\left|\hat{f^{*}}(\xi)\right|^{2} d \xi,
\end{aligned}
$$

where

$$
(g * h)(x)=\int_{\mathbb{R}^{n}} g(x-y) h(y) d y .
$$


Inequality (16) is due to the classical Riesz-Sobolev lemma, or see also Beckner's symmetrization lemma [3]:

Lemma 2. Let $\mathbf{M}$ denote $\mathbb{R}^{n}, \mathbf{S}^{n}$ or $\mathbf{H}^{n}$ (real hyperbolic space) with a symmetric decreasing rearrangement defined in terms of geodesic distance. Let $\mathbf{K}$ be a monotonically decreasing function and let $d(x, y)$ denote the distance between the points $x$ and $y$ in the symmetric space $\mathbf{M}$. Then

$$
\int_{\mathbf{M} \times \mathbf{M}} f(x) \mathbf{K}[d(x, y)] g(y) d x d y \leq \int_{\mathbf{M} \times \mathbf{M}} f^{*}(x) \mathbf{K}[d(x, y)] g^{*}(y) d x d y,
$$

where $f$ and $g$ are nonnegative measurable functions with $f^{*}$ and $g^{*}$ denoting their respective equimeasurable, geodesically decreasing rearrangements on $\mathbf{M}, d x$ denotes the measure invariant under the group action on $\mathbf{M}$ and the integrand on the left is

in $L^{1}(\mathbf{M} \times \mathbf{M})$. If $\mathbf{K}$ is strictly decreasing, then the above inequality is strict unless $f(x)=f^{*}(\tau x)$ and $g(x)=g^{*}(\tau x)$ almost everywhere for $\tau x$ a "translate" of $x$.

\section{ACKNOWLEDGEMENT}

I would like to thank the referee for thoughtful remarks and for pointing out a valuable reference.

\section{REFERENCES}

[1] T. Aubin, Nonlinear analysis on manifolds: Monge-Ampère equations, Grundlehren der mathematischen Wissenschaften, Band 252, Springer-Verlag, New York, 1982. MR 85j:58002

[2] W. Beckner, Inequalities in Fourier analysis, Ann. Math., 102: 159-182, 1975. MR 52:6317

[3] W. Beckner, Sobolev inequalities, the Poisson semigroup, and analysis on the sphere $\mathbf{S}^{n}$, Proc. Natl. Acad. Sci., 89: 4816-4819, 1992. MR 93d:26018

[4] W. Beckner, Sharp Sobolev inequalities on the sphere and the Moser-Trudinger inequality, Ann. Math., 138: 213-242, 1993. MR 94m:58232

[5] W. Beckner, Geometric inequalities in Fourier analysis, Essays on Fourier Analysis in honor of Elias M. Stein, Princeton University Press, Princeton, NJ, 36-68, 1995. MR 95m:42004

[6] W. Beckner, Pitt's inequalities and the uncertainty principle, Proc. Amer. Math. Soc., 123: 1897-1905, 1995. MR 95g:42021

[7] W. Beckner, Sharp inequalities and geometric manifolds, J. Fourier Anal. Appl., 3: 825-836, 1997. MR 2000c:58059]

[8] W. Beckner, Geometric asymptotics and the logarithmic Sobolev inequality, Forum Math., 11: 105-137, 1999. MR 2000a:46049

[9] W. Beckner and M. Pearson, On sharp Sobolev embedding and the logarithmic Sobolev inequality, Bull. London Math. Soc., 30: 80-84, 1998. MR 98k:46048

[10] E. A. Carlen, Superadditivity of Fisher's information and logarithmic Sobolev inequalities, J. Funct. Anal., 101: 194-211, 1991. MR 92k:94006

[11] J. F. Escobar, Sharp constant in a Sobolev trace inequality, Indiana Math. J., 37 : 687-698, 1988. MR 90a:46071

[12] J. F. Escobar, The Yamabe problem on manifolds with boundary, J. Differential Geom., 35: 21-84, 1992. MR 93b:53030

[13] L. Gross, Logarithmic Sobolev inequalities, Amer. J. Math., 97: 1061-1083, 1975. MR 54:8263

[14] I. I. Hirschman, Jr., A note on entropy, Amer. J. Math., 79: 152-156, 1957. MR 19:622i

[15] B. Kawohl, Rearrangements and convexity of level sets in PDE, Lecture Notes in Mathematics, no. 1150, Springer-Verlag, Berlin, 1985. MR 87a:35001

[16] E. H. Lieb and M. Loss, Analysis, Graduate Studies in Mathematics, Vol. 14, American Mathematical Society, Providence, RI, 1997. MR 98b:00004

[17] A. J. Stam, Some inequalities satisfied by the quantities of information of Fisher and Shannon, Information and Control 2, 101-112, 1959. MR 21:7813

[18] E. M. Stein, Singular integrals and differentiability properties of functions, Princeton University Press, Princeton, NJ, 1970. MR 44:7280 
[19] E. M. Stein and G. Weiss, Introduction to Fourier analysis on Euclidean spaces, Princeton University Press, Princeton, NJ, 1971. MR 46:4102

[20] F. B. Weissler, Logarithmic Sobolev inequalities for the heat-diffusion semigroup, Trans. Amer. Math. Soc., 237: 255-269, 1978. MR 80b:47057

[21] E. T. Whittaker and G. N. Watson, A course of modern analysis, Cambridge University Press, Cambridge, 1927. MR 97k:01072

Department of Mathematics, University of Texas at Austin, Austin, Texas 78712-1082 E-mail address: ypark@math.utexas.edu

Current address: Department of Applied Mathematics, Sejong University, 98 Kunja-dong, Kwangjin-ku, Seoul, South Korea 143-747

E-mail address: 0_park@hanmail.net 